\title{
Convulsão febril: aspectos clínicos e terapêuticos. Artigo de revisão
}

\section{Febrile seizures: clinical and therapeutic aspects. Review article}

\author{
Sarah Pinheiro de Alencar ${ }^{1}$. \\ 1 Residente de Pediatria do Hospital Universitário Walter Cantídio - UFC.
}

\section{RESUMO}

Convulsões febris são uma manifestação epiléptica benigna da infância que ocorre entre 3 meses e 5 anos de idade e que afeta um número estimado de $2-5 \%$ das crianças. As convulsões febris simples têm um prognóstico benigno em quase todos os casos e não necessitam de uma extensa investigação diagnóstica. As convulsões febris complexas necessitam de uma avaliação clínica mais detalhada e exames complementares podem ser indicados devido ao maior risco de causas subjacentes detectáveis e um discreto maior risco para o desenvolvimento posterior de epilepsia. As convulsões febris geralmente não têm efeitos negativos importantes no desenvolvimento cognitivo e motor. O uso da profilaxia com anticonvulsivantes tem controvérsias na literatura, porém não são rotineiramente recomendados tanto em crises simples, como nas complexas, uma vez que seus efeitos colaterais superam os benefícios dessa prática. Nesta revisão, serão abordados os aspectos clínicos e epidemiológicos das convulsões febris, além das considerações acerca do seu tratamento.

Palavras-chave: Convulsões. Febre. Crianças. Epilepsia.

\section{ABSTRACT}

Febrile seizures are benign epileptic manifestation of childhood that occurs between 3 months and 5 years of age and affects an estimated $2-5 \%$ of children. The simple febrile seizures have a benign prognosis in almost all cases, and do not require an extensive diagnostic investigation. Complex febrile seizures require a more detailed clinical evaluation and laboratory tests may be indicated because of the increased risk of underlying causes detectable and a slight increased risk for later development of epilepsy. Febrile seizures usually do not have significant negative effects on cognitive and motor development. The use of prophylactic anticonvulsants is controversial in the literature, but is not routinely recommended both simple crises, as in the complex, since its side effects outweigh the benefits of this practice. In this review, clinical and epidemiological aspects of febrile seizures will be addressed, in addition to considerations about their treatment.

Keywords: Seizures. Fever. Children. Epilepsy.

Autor correspondente: Sarah Pinheiro de Alencar. Avenida Barão de Studart, 2445, apto 303, Joaquim Távora, Fortaleza, Ceará. CEP:60120002. Telefone: +55 85 99403680. E-mail: alencarsarah@hotmail.com.

Conflito de interesses: Não há qualquer conflito de interesses por parte de qualquer um dos autores.

Recebido em: 30 Mar 2015; Revisado em: 05 Mai 2015; Aceito em: 07 Mai 2015. 


\section{INTRODUÇÃO}

Convulsão febril (CF) é definida como crise convulsiva acompanhada por febre (temperatura maior ou igual a $38^{\circ} \mathrm{C}$ por qualquer método de medida) que ocorre em crianças de 6 meses a 60 meses de idade sem evidência de infecção ou inflamação do sistema nervoso central, alteração metabólica e sem história prévia de crise convulsiva. ${ }^{1} \mathrm{CF}$ s não devem ser confundidas com epilepsia, que se caracteriza por crises epilépticas afebris recorrentes. ${ }^{2}$

As crises convulsivas febris são um dos problemas neurológicos mais comuns da infância e estima-se que $2 \%$ a $5 \%$ das crianças menores de cinco anos de idade apresentarão pelo menos um episódio de convulsão em vigência de febre na vida. ${ }^{3}$

\section{MÉTODOS}

Foi realizada revisão da literatura nacional e internacional utilizando o banco de dados MEDLINE (PUBMED), sendo selecionados artigos publicados nos últimos quinze anos, abordando a doença convulsão febril com ênfase em suas características clínicas, epidemiológicas e terapêuticas. Foi utilizada na busca a intersecção dos seguintes termos de pesquisa (palavras-chaves): 1) convulsão; 2) epilepsia; 3) febre; 4) criança.

A pesquisa bibliográfica incluiu artigos originais, artigos de revisão e diretrizes escritos nas línguas inglesa, espanhola e portuguesa.

Foram utilizados 18 artigos, sendo 8 nacionais e 10 internacionais, disponíveis online em texto completo. Para a seleção das fontes, foram consideradas como critério de inclusão aquelas bibliografias que abordassem a convulsão febril e consequentemente a temática relacionada a suas características clínicas, epidemiológicas e terapêuticas, e foram excluídas aquelas que não contemplassem a temática acima, ou não abordassem a faixa etária pediátrica.

\section{Fisiopatologia}

Estudos clínicos e experimentais demonstram que o cérebro imaturo apresenta maior susceptibilidade a convulsões. ${ }^{2}$ Os mecanismos fisiopatológicos para a convulsão febril ainda não foram claramente estabelecidos. Penfield e Erikson acreditam que a condição de imaturidade do cérebro, a falta de mielina, a diferença de permeabilidade celular e a atividade elétrica do cérebro da criança são algumas das razões que tornam as crianças mais susceptíveis a convulsões febris do que os adultos. ${ }^{4}$

A verificação de que algumas famílias são mais suscetíveis a apresentarem convulsão febril é notória e já foram descritos os loci cromossômicos de algumas delas. ${ }^{1}$ Em um estudo foi observado que os filhos de pais que foram acometidos por CF tiveram um risco 4,4 vezes maior do que a população geral de também apresentarem crise febril, e esse risco pode ser ainda maior ( 8 vezes) quando a mãe é acometida. Quando irmãos têm $\mathrm{CF}$, o risco é 3,5 vezes maior do que para a população em geral. ${ }^{4}$ No entanto, ainda não foi identificado um modelo de transmissão que atenda a todos os casos de convulsão febril. A maioria dos estudos sugere herança autossômica dominante, com baixa penetrância e expressão variável, ou herança poligênica. ${ }^{5}$

\section{Etiopatogenia}

A etiologia do processo infeccioso não parece ser determinante para a crise febril. Entretanto, a presença de febre é essencial, embora não se conheça seu mecanismo de ação. ${ }^{3}$

Os vírus são normalmente mais envolvidos com convulsões febris, por serem os mais prevalentes na comunidade. É comum a associação com o exantema súbito (Roseolainfantum), e menos frequentemente com o sarampo. ${ }^{4}$ O tipo de doença desencadeante da febre mais frequente é a doença respiratória. Alguns estudos observaram sazonalidade na incidência das convulsões febris, sendo maior no outono, quando a taxa de doenças respiratórias é maior. ${ }^{6}$

\section{Características clínicas}

De modo geral, as convulsões febris são tônico-clônicas generalizadas, de curta duração, únicas e precoces em uma mesma doença febril. Essas características definem as convulsões febris simples ou típicas. Crises com duração maior que dez minutos, parciais, que se repetem durante o mesmo episódio febril, e acompanhadas por sinais neurológicos transitórios são denominadas atípicas ou complexas. ${ }^{5}$ Aproximadamente $80 \%$ das crises convulsivas febris são do tipo simples. ${ }^{2}$

Recentemente foi sugerida a duração de 5 minutos do episódio convulsivo como o limite para uma intervenção terapêutica ativa. Esta observação foi feita porque crises que duram mais de $5 \mathrm{~min}$, muitas vezes não param espontaneamente e tem um maior potencial para induzir permanente lesão neuronal e ou resistência a drogas. ${ }^{7}$

O exame neurológico imediatamente após a crise pode mostrar sonolência, ataxia, e eventualmente sinais focais, se a crise for do tipo complexo; após algumas horas, geralmente é normal, exceto se a criança estiver sedada. Nos pacientes com anomalias estruturais do SNC, que aumentam o risco de recorrência da $\mathrm{CF}$, o exame neurológico pode manter-se alterado após a crise epiléptica. ${ }^{6}$

\section{Risco de recorrência e epilepsia}

De um modo geral, a maioria das crianças com CF apresenta um único episódio durante a vida. ${ }^{1} \mathrm{O}$ risco de recorrência da crise febril varia em torno de $30 \%$,sendo mais comum entre seis meses e três anos de idade. Além disso, a chance de recorrência diminui com o passar do tempo. Segundo Nelson e Ellenberg, 50\% das crianças apresentam a segunda crise febril até seis meses após a primeira, 75\% em um ano e $90 \%$ em dois anos. ${ }^{3}$

Os fatores de risco inerentes à criança para recorrência de 
convulsão são: idade precoce da primeira crise (considerada menor que 18 meses), sexo masculino, antecedente familiar de CF ou epilepsia, e anormalidade do desenvolvimento neuropsicomotor. Os fatores de risco de recorrência associados às características do evento convulsivo são: crises focais, duração prolongada, e recorrência de crise epiléptica no mesmo episódio de doença desencadeante. ${ }^{6}$

Este risco de crises recorrentes nas crianças previamente saudáveis foi estimado em um estudo colaborativo que utilizou os dados individuais de cinco estudos de follow-up com definições semelhantes de convulsões febris e fatores de risco (Offringa, 1994). De um total de 2.496 crianças com 1.410 episódios de crises recorrentes neste estudo, 32\% tinham pelo menos um, $15 \%$ tiveram pelo menos dois e $7 \%$ tinham três ou mais crises recorrentes após uma primeira convulsão febril. O risco de crises recorrentes foi maior entre as idades de 12 e 24 meses. $^{8}$

A epilepsia é doença reconhecida para crianças com quadro clínico de crise(s) epiléptica(s), manifestação transitória de sinais e sintomas devidos à atividade neuronal excessiva ou síncrona de neurônios cerebrais, sem fator desencadeante em, ao menos, um episódio. A febre pode ser o fator desencadeante de alguns episódios, e por isto alguns pacientes com epilepsia podem ser inicialmente diagnosticados como acometidos por $\mathrm{CF}{ }^{6}$

No que se refere ao risco de epilepsia em crianças que tiveram convulsão febril, estudos epidemiológicos demonstram que o risco é bastante baixo: 2-10\%. No entanto, pode aumentar na presença de determinados fatores. Anormalidades do desenvolvimento neurológico, história familiar de epilepsia e crises febris complexas são relatados como os preditores primários de persistência de convulsões nessas crianças. A relação entre as características da convulsão febril, bem como tipo de epilepsia subsequente, no entanto, ainda é uma questão de debate. ${ }^{9}$

Quando são analisadas as séries de epilepsia de lobo temporal (ELT), por outro lado, a história prévia de CF é frequente e, inclusive, pode ser um fator de bom prognóstico cirúrgico. A esclerose mesialhipocampal (EMH) é a causa mais frequente de ELT em adultos e, discute-se na literatura, se a EMH é causa ou consequência de $\mathrm{CF}{ }^{2}$

\section{Diagnóstico}

O diagnóstico das crises febris é clínico, através de anamnese e exame físico cuidadosos, devendo sempre classificar a crise em simples ou complexa e identificar o possível foco da febre. ${ }^{3}$

Ao receber uma criança com quadro de convulsão em vigência de febre, ou com esse relato pelos pais, deve-se solicitar que descrevam com detalhes a crise, e pesquisar de imediato a presença de sinais meníngeos, bem como realizar o exame da fontanela, visando a descartar acometimento do sistema nervoso central. ${ }^{2}$

É necessária uma história adequada, pesquisando-se todas as intercorrências no período da gestação, parto ou a presença de qualquer doença sistêmica concomitante como, por exemplo, cardiopatias, coagulopatias ou distúrbios hidroeletrolíticos. Devemos insistentemente questionar antecedentes de uso de drogas, traumas ou outras patologias anteriores. $^{10}$

Com relação ao diagnóstico laboratorial, não são recomendados exames laboratoriais para crise febril simples; no caso de crises complexas, realizar se não for identificado o foco da febre. ${ }^{11}$

Considerar fortemente a punção lombar em crianças abaixo de 12 meses após a primeira $\mathrm{CF}$, nas quais as manifestações de infecção do SNC podem não estar presentes, e naquelas entre 12 e 18 meses de vida, nas quais estas manifestações podem ser incertas. Em pacientes acima de 18 meses com primeiro episódio de CF a punção lombar não deve ser realizada rotineiramente, mas sim mediante a observação clínica de sinais e sintomas sugestivos de infecção central. ${ }^{11}$

Em relação aos achados do eletroencefalograma (EEG), não existe evidência de que um EEG pode ser utilizado para prever se uma criança irá desenvolver epilepsia após uma convulsão febril simples. Podemos considerar a realização de EEG se mais de uma característica complexa está presente. ${ }^{12}$ Alguns autores verificaram que anormalidades encontradas no eletroencefalograma são prognósticas, enquanto outros discordam. ${ }^{6}$

Exames de neuroimagem não são indicados após uma convulsão febril simples, mas podem ser considerados quando houver aspectos clínicos de uma doença neurológica, por exemplo, micro/macrocefalia, anormalidades neurocutâneas, déficit neurológico pré-existente, persistência de déficit neurológico pós-ictal, ou quando há crises febris complexas recorrentes, e particularmente em caso de dúvida se as crises são febris. ${ }^{11}$ Para essas crianças, considerar a realização de ressonância magnética do cérebro, pois tem maior sensibilidade do que a tomografia computadorizada para avaliar a presença de uma anormalidade estrutural que justifique a crise. ${ }^{12}$

\section{Tratamento}

$\mathrm{O}$ tratamento da crise febril engloba fase aguda, profilaxia e orientação aos familiares. A maior parte das crises termina antes dos pacientes chegarem ao pronto-atendimento e o médico na maioria das vezes avalia a criança já no período pós-ictal. ${ }^{3,11} \mathrm{O}$ tratamento da crise convulsiva febril na fase aguda deve ser feito como o de qualquer crise epiléptica. A sequência de atendimento de um quadro de urgência (avaliação de vias aéreas, ventilação e circulação), antes da infusão de medicação específica,deve ser respeitada. ${ }^{13,10}$

Para crianças que tenham experimentado uma crise convulsiva febril, os potenciais efeitos adversos que, teoricamente, poderiam ser alterados com terapêutica eficaz seriam: 1- declínio do QI; 2- o aumento do risco de epilepsia; 3- risco de convulsões febris recorrentes; e 4- morte. Nenhuma dessas consequências foi observada em crianças que tiveram crise febril simples, exceto naquelas crianças que tinham anormalidades neurológicas antes de sua primeira 
crise. $^{14}$

A classe de drogas de primeira linha consiste nos benzodiazepínicos, como o diazepam $(0,2$ e $0,3 \mathrm{mg} / \mathrm{kg}$, via endovenosa ou retal podendo ser repetido até dose cumulativa de $5 \mathrm{mg}$ ) e o midazolam ( 0,2 a $0,7 \mathrm{mg} / \mathrm{kg}$, via endovenosa, intramuscular ou retal).,15

Em relação ao uso de antitérmicos, por mais precoce e eficaz que seja, não previne a recorrência das crises. ${ }^{14}$ Utilização intensa e rotineira de agentes antipiréticos não tem sido mais eficaz na redução da incidência de convulsões febris recorrentes que o uso intermitente dos antipiréticos, quando um episódio febril é percebido. ${ }^{16}$

Dadas as características benignas das convulsões febris simples e os potenciais efeitos adversos da terapia anticonvulsivante, não se recomenda tratamento profilático para prevenção de recorrência de crises. $^{6}$ Nenhuma medicação tem demonstrado reduzir o risco de um ataque afebril após uma convulsão febril simples. ${ }^{16}$

\section{Profilaxia contínua}

Por muitos anos foi usado o fenobarbital (3 a $5 \mathrm{mg} / \mathrm{kg} / \mathrm{dia}$ ), que foi considerado eficaz em alguns estudos na prevenção de novas crises febris, no entanto para esse efeito seus níveis séricos precisam estar em concentrações terapêuticas, o que foi atribuído a uma série de efeitos colaterais inconvenientes, que podem ser graves o suficiente para suspensão do tratamento. ${ }^{17}$ Sintomas como hiperatividade, irritabilidade, hipersensibilidade e distúrbio do sono são comuns. Deve ser analisado ainda o aparente risco de decréscimo do quociente de inteligência (QI), conforme demonstrado em um estudo randomizado. $^{2}$

O ácido valpróico (15 a $60 \mathrm{mg} / \mathrm{kg} / \mathrm{dia}$ ) pareceu ser pelo menos tão eficaz na prevenção de convulsões febris simples recorrentes quanto fenobarbital e significativamente mais eficaz que placebo. ${ }^{14} \mathrm{O}$ ácido valpróico apresenta risco de hepatite fulminante, efeito colateral raro, mas que limita o seu uso particularmente em crianças pequenas, para uma entidade tão benigna como a convulsão febril. Além disso, outros efeitos adversos com o uso que limitam o seu uso são intolerância gástrica, ganho de peso e toxicidade renal. ${ }^{2,16}$

Outras drogas também foram testadas, mas ou não preveniram a recorrência das crises (fenitoína) ou não foram mais eficazes que o fenobarbital (carbamazepina). ${ }^{3}$

\section{Profilaxia intermitente}

Como forma de minimizar os efeitos colaterais dessas drogas em profilaxia contínua, foi proposta a profilaxia intermitente com anticonvulsivantes, sendo os mais estudados os benzodiazepínicos. ${ }^{15}$ Eles apresentam boa tolerabilidade, sendo que $20 \%$ a $30 \%$ dos pacientes apresentam efeitos colaterais de leve a moderada intensidade, que são transitórios e não impossibilitam o uso da medicação. Recomenda-se o uso do diazepam via oral na dose de 0,5 a1 $\mathrm{mg} / \mathrm{kg} / \mathrm{dia}$, entretanto a orientação é que seu uso seja restrito aos casos com algum dos fatores de risco já citados para recorrência, e que a família seja orientada para que a profilaxia seja iniciada a qualquer sinal de adoecimento da criança. A medicação deverá ser suspensa aproximadamente 24 horas após o último pico febril. ${ }^{2,18}$

O midazolam bucal parece ser tão eficaz quanto o diazepam na redução do tempo de crise. Num estudo, o midazolam intranasal foi mais eficaz que o diazepam retal em encerrar o estado epiléptico em crianças. No grupo diazepam, a crise de $60 \%$ dos pacientes parou aos 10 minutos, enquanto que, $87 \%$ das convulsões pararam no grupo do midazolam intranasal em 10 minutos. ${ }^{4}$ Outro benzodiazepínico que apresenta eficácia e tolerabilidade semelhantes ao diazepam é o clobazam, porém são necessários mais estudos para determinar sua eficácia e seus efeitos adversos. ${ }^{8}$

O uso do fenobarbital na época da febre foi provado ser ineficaz, provavelmente por causa do atraso na obtenção de níveis séricos e teciduais apropriados. ${ }^{7}$

Em conclusão, não existem fortes recomendações para fazer uso de profilaxia medicamentosa na convulsão febril ou com fenobarbital contínuo ou com diazepam intermitente, por causa da heterogeneidade dos estudos existentes, mas há uma evidência de benefício potencial com o tratamento com ambas as drogas, embora não seja possível a concluir qual a melhor terapia. ${ }^{18}$

\section{Orientações aos familiares}

Devemos alertar quanto à benignidade do quadro, à possibilidade de recorrência e ao risco levemente aumentado de desenvolver epilepsia no futuro, mas sempre com o objetivo de que a criança leve uma vida normal.,

Durante a ocorrência da crise, algumas das medidas a serem orientadas aos pais são que eles devem tentar manter a calma, proteger contra traumas durante o período ictal, impedir que se coloque algum objeto na boca da criança, posicionar lateralmente o paciente para prevenir aspiração de saliva e monitorar o tempo de crise. ${ }^{3,13}$

\section{CONCLUSÃO}

As crises convulsivas febris são um problema neurológico comum na infância, mas de baixa morbidade e recorrência pouco frequente. Na maioria dos casos, as crises febris são benignas e a grande maioria das crianças apresenta desenvolvimento normal após esse evento, no entanto essas crises levam a um grande sofrimento para o paciente e sua família. Para reduzir a ansiedade dos pais, a história natural dessa patologia deve ser bem explicada.

A profilaxia secundária com anticonvulsivantes atualmente não tem suporte na literatura no caso de crises febris simples, mas no caso de crises febris complexas a profilaxia secundária intermitente ou contínua pode ser considerada. A decisão do tratamento deve ser individualizada e resultar de adequado julgamento e da experiência do médico. Considerar a certeza do diagnóstico, os potenciais riscos e benefícios, as chances de recorrência, a probabilidade de adesão ao tratamento e o desejo do paciente e/ou de seus pais ou responsáveis. 


\section{REFERÊNCIAS}

1. Durante ME, Cancelier AC. Prevalência de internações por convulsões febris em serviço de pediatria no sul do país: análise dos fatores associados. Arq Catarin Med. 2007;36(4):7-11.

2. Guerreiro MM. Tratamento das crises febris. J Pediatr. 2002;78 Supl 1:S9-13.

3. Siqueira LF. Atualização no diagnóstico e tratamento das crises epilépticas febris. Rev Assoc Med Bras. 2010;56(4):489-92.

4. Ghofrani MD. Febrile convulsion: another look at an old subject. Iran J Child Neurol. 2006;1(1):5-9.

5. Campos D Jr, Burns DA, Lopez FA. Tratado de pediatria. 3. ed Barueri: Sociedade Brasileira de Pediatria; 2014.

6. Guilhoto LM, Felgueira M, Lioi MC, Lioi MI. Ensaio: o pediatra frente à crise epiléptica febril. Pediatria (São Paulo). 2005;27(2):103-13

7. Mastrangelo M, Midulla F, Moretti C. Actual insights into the clinical management of febrile seizures. Eur $\mathrm{J}$ Pediatr. 2014;173(8):977-82.

8. Offringa M, Newton R. Prophylactic drug management for febrile seizures in children. Cochrane Database Syst Rev. 2012 Apr $18 ;(4):[1$ p.]

9. Saltik S, Angay A, Ozkara C, Demirbilek V, Dervant A. A retrospective analysis of patients with febrile seizures followed by epilepsy. Seizure. 2003;12(4):211-6.
10. Casella EB, Mangia CM. Abordagem da crise convulsiva aguda e estado de mal epiléptico em crianças. J Pediatr (Rio J). 1999;75 Supl 2:S197-S206.

11. Guidelines and Protocols Advisory Committee (CA). Febrile Seizures. Ann Emerg Med. 2003;41:215-22.

12. Seinfeld S, Pellock JM. Recent research on febrile seizures: a review. J Neurol Neurophysiol. 2013;4(4):1-6.

13. Recalde LR, Doldán ME, Sostoa G, Aldana A, Lezcano M. Convulsíon febril. Pediatria. (Asunción). 2011;38(1);63-7.

14. Steering Committee on Quality Improvement and Management, Subcommittee on Febrile Seizures American Academy of Pediatrics. Febrile Seizures: Clinical Practice Guideline for the long-term management of the child with simple febrile seizures. Pediatrics. 2008;121(6):1281-6.

15. Maia HS Filho. Abordagem das crises epilépticas na emergência pediátrica. Revista de Pediatria SOPERJ. 2012;13(2):29-34.

16. Millar JS. Evaluation and treatment of the child with febrile seizure. Am Fam Physician. 2006;73(10):1761-4.

17. Brophy GM, Bell R, Claassen J, Alldredge B, Bleck TP, Glauser $\mathrm{T}$, et al. Guidelines for the evaluation and management of status epilepticus. Neurocrit Care. 2012;17(1):3-23.

18. Masuko AH, Castro AA, Santos GR, Atallah AN, Prado LB, Carvalho LB, et al. Intermittent diazepam and continuous phenobarbital to treat recurrence of febrile seizures: a systematic review with meta-analysis. Arq Neuropsiquiatr. 2003;61(4):897901 .

\section{Como citar:}

Alencar SP. Convulsão febril: aspectos clínicos e terapêuticos. Artigo de revisão. Rev Med UFC. 2015 jan-jun;55(1):38-42. 